\title{
Evaluation of demand for different trip purposes under various congestion pricing scenarios
}

\begin{abstract}
Cordon pricing is an effective policy to alleviate congestion and manage travel demand in city centres. However, different responses among road users to certain policies are inevitable. This paper presents a study evaluating the possible consequences of cordon and parking pricing on road users with different trip purposes, i.e., workers and non-workers. Different models have been developed and calibrated with data from the central business district of Mashhad, Iran, using revealed and stated preference methods. The analysis of the interviewee responses to the hypothetical situations shows that workers comprise the dominant proportion using each mode of travel to the Mashhad CBD. The commuters were provided with alternative choices, and their preferences were examined using multinomial logit. The results show that the cost-based policy such as parking and cordon pricing is good to reduce congestion as it affects workers, but the impact is more evident for non-workers travelling to the CBD. Furthermore, the model suggests that workers are more likely to shift mode in response to changes in travel time. The model was also developed to obtain different willingness-to-pay measures, which indicate that workers have a greater willingness to pay for parking and cordon pricing than non-workers.
\end{abstract}

Keyword: Congestion pricing, Parking cost, Stated-preference, Trip purpose 